Supporting Information

Magnetic Doping Induced Superconductivity-to-

Incommensurate Density Waves Transition in 2D Ultrathin Cr-

\title{
Doped $\mathrm{Mo}_{2} \mathrm{C}$ Crystal
}

Shaojian $\mathrm{Li}^{1 \dagger}$, Zongyuan Zhang ${ }^{1,7 \dagger}$, Chuan $\mathrm{Xu}^{2 \dagger}$, Zhen $\mathrm{Liu}^{3 \dagger}$, Xiaorui Chen ${ }^{4}$, Qi

Bian $^{1}$, Habakubaho Gedeon ${ }^{1}$, Zhibin Shao ${ }^{4}$, Lijun Liu ${ }^{1}$, Zhibo Liu ${ }^{2}$, Ning Kang ${ }^{3 *}$, Hui-Ming Cheng ${ }^{2,5,6}$, Wencai Ren ${ }^{2,5^{*}}$ and Minghu Pan ${ }^{1,4 *}$

Optical images of $\mathrm{Cr}$-doped $\mathrm{Mo}_{2} \mathrm{C}$ crystal with different shapes and sizes
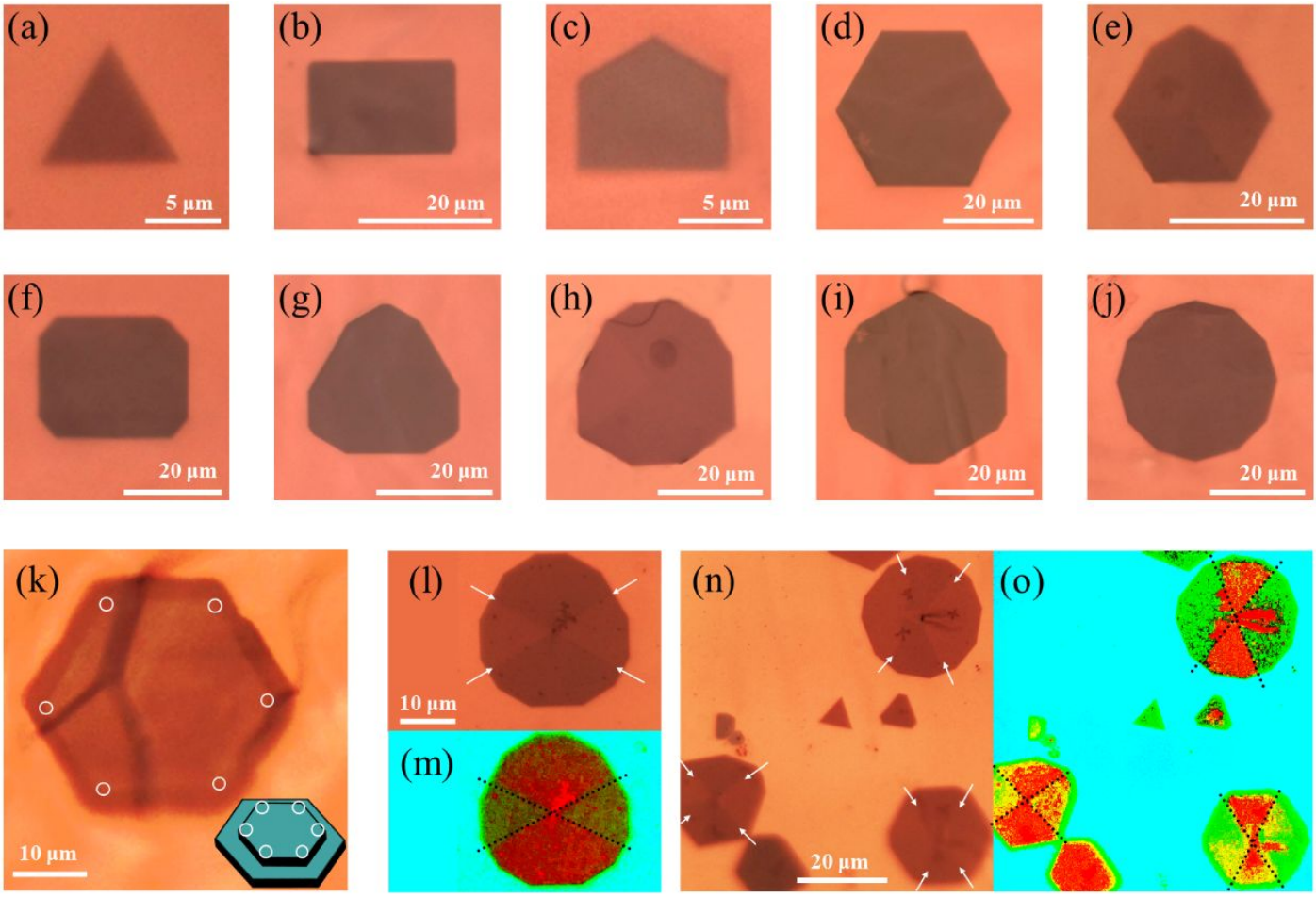

Figue S1.Optical images of $\mathrm{Cr}$-doped $\mathrm{Mo}_{2} \mathrm{C}$ crystal with different shapes and sizes (a-j). (k-o) High contrast optical images show different thicknesses on the top of $\mathrm{Mo}_{2} \mathrm{C}$ microcrystal.

DFT calculations of electronic band structures for various $\mathrm{Cr}$-doping level. 


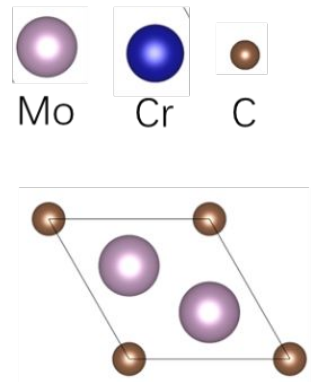

$\mathrm{Mo}_{2} \mathrm{C} 1 \times 1 \cup \mathrm{C}$

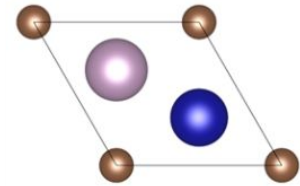

$1 \times 1$ UC Cr: $50 \%$

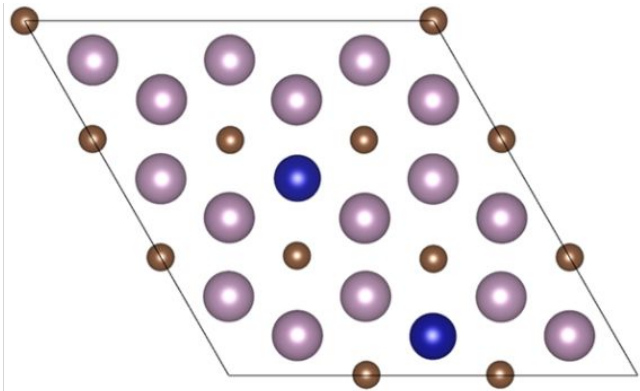

$3 \times 3$ UC Cr: $11.1 \%$

Figure S2 Atomic models for DFT calculation of pristine $\mathrm{Mo}_{2} \mathrm{C}, 50 \%$ and $11 \%$ Cr-doping $\mathrm{Mo}_{2} \mathrm{C}$ monolayers.
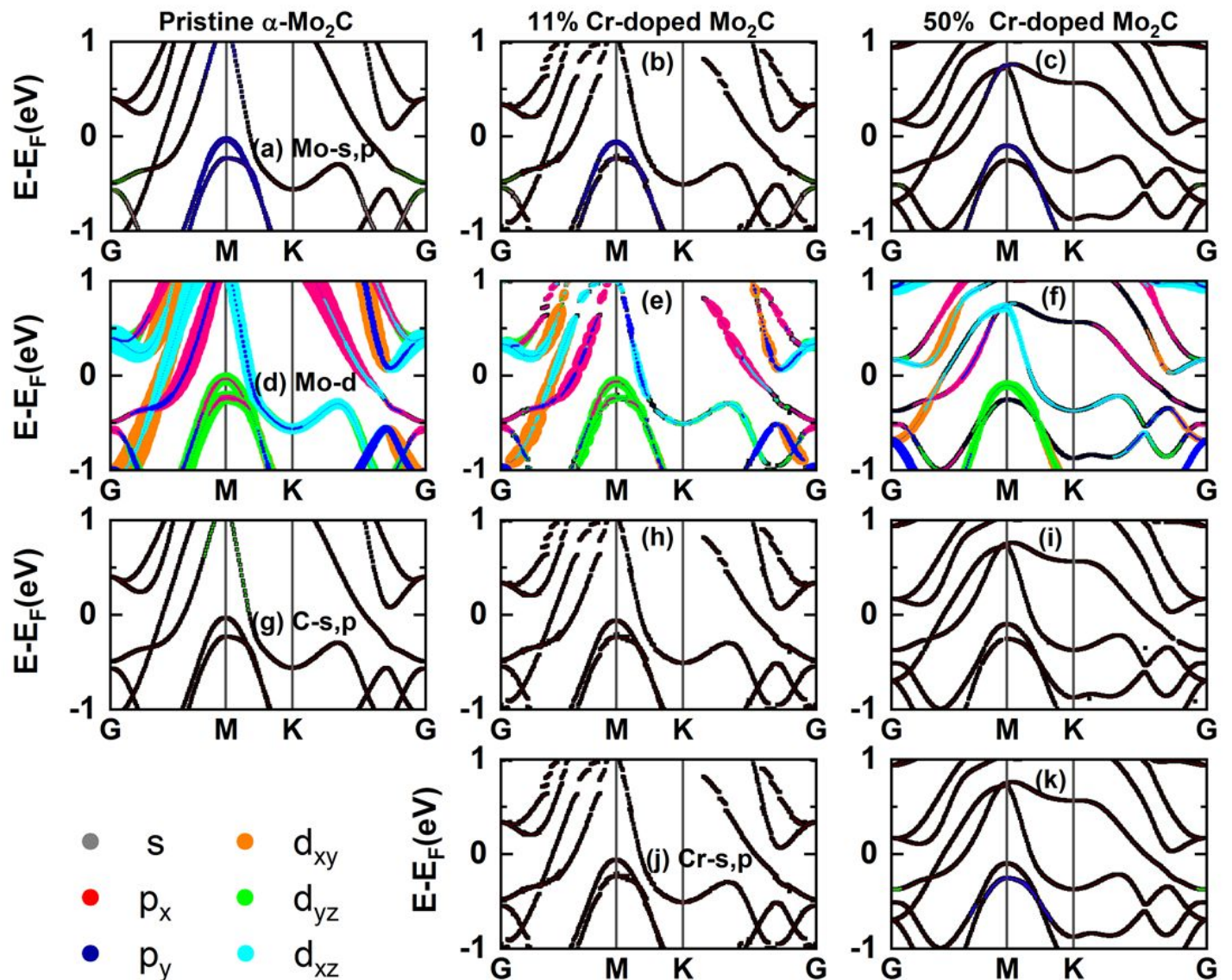

- $s \cdot d_{x y}$

- $p_{x} \bullet d_{y z}$

- $p_{y} \bullet d_{x z}$

- $p_{z}$

$$
\text { - } d_{z^{2}}
$$
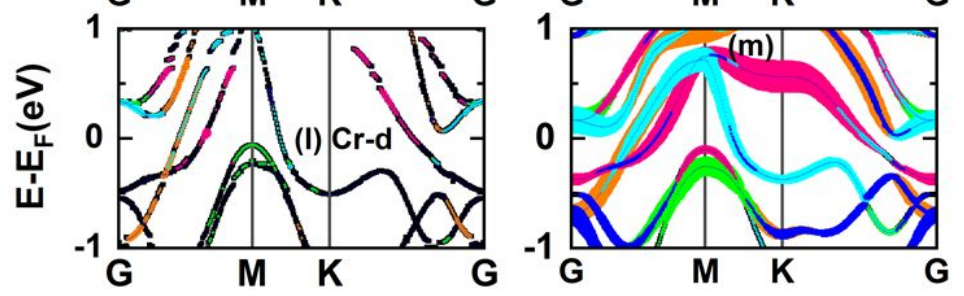

Figure S3 Calculated band structures of pristine $\mathrm{Mo}_{2} \mathrm{C}, 50 \%$ and $11 \% \mathrm{Cr}$-doping $\mathrm{Mo}_{2} \mathrm{C}$ monolayers. The colors denote different atomic orbitals of $\mathrm{Mo}, \mathrm{C}$ and $\mathrm{Cr}$ atoms. 
The thicknesses of bands denote the projected DOS.

\section{Characterization of $\mathrm{Cr}$-doped $\mathrm{Mo}_{2} \mathrm{C}$ samples with high-resolution scanning transmission electron microscopy (HR-STEM)}

The atomic-level HR-STEM image confirms that such $\mathrm{Mo}_{2} \mathrm{C}$ with 9.4 at $\% \mathrm{Cr}$ still has the same crystal structure as the pristine $\alpha-\mathrm{Mo}_{2} \mathrm{C}$ crystal (Fig. S4e). Moreover, the crystal has a highly crystalline quality. The similar brightness of each atomic column suggests no element segregation and second phase even at such $\mathrm{Cr}$ doping level (Fig. $\mathrm{S} 4 \mathrm{e}$ ). The sites of $\mathrm{Cr}$ are completely overlapped with those of Mo, which means $\mathrm{Cr}$ atoms substitute a part of $\mathrm{Mo}$ atoms in $\mathrm{Mo}_{2} \mathrm{C}$ lattice. The unchanged lattice constant is attributed to the similar size of $\mathrm{Cr}$ and Mo atoms. The EDS mappings confirm the uniform distribution of $\mathrm{Cr}$ in $\mathrm{Mo}_{2} \mathrm{C}$ crystals (Fig. S4b-d).
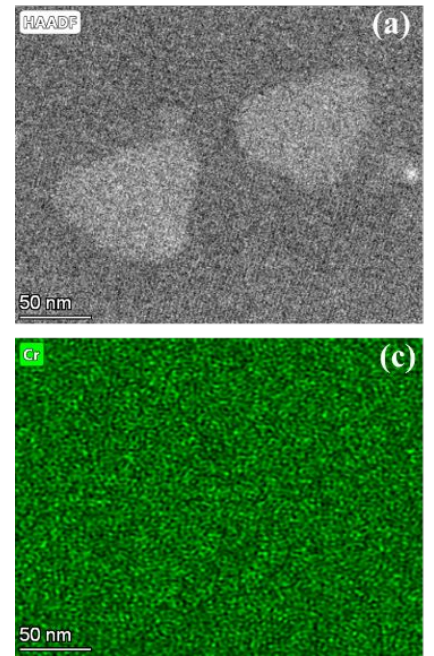
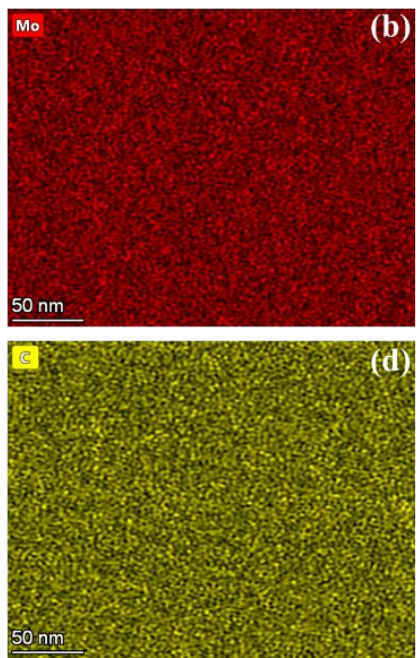

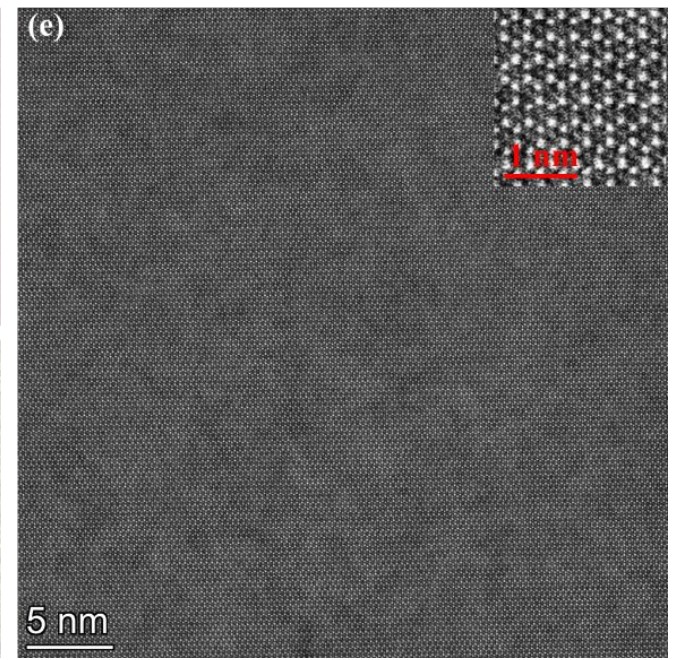

Figure S4 HR-STEM image of a 2D $\mathrm{Mo}_{2} \mathrm{C}$ crystal with 9.4 at\% $\mathrm{Cr}$. (a-d) The image of a selected area (a), the corresponding EDS mappings of Mo-Ka (b), $\mathrm{Cr}-\mathrm{K}$ (c) and C (d). (e) Atomic-level STEM image.

Different oriented modulations and the domain-like structure in large scaled

\section{STM images}



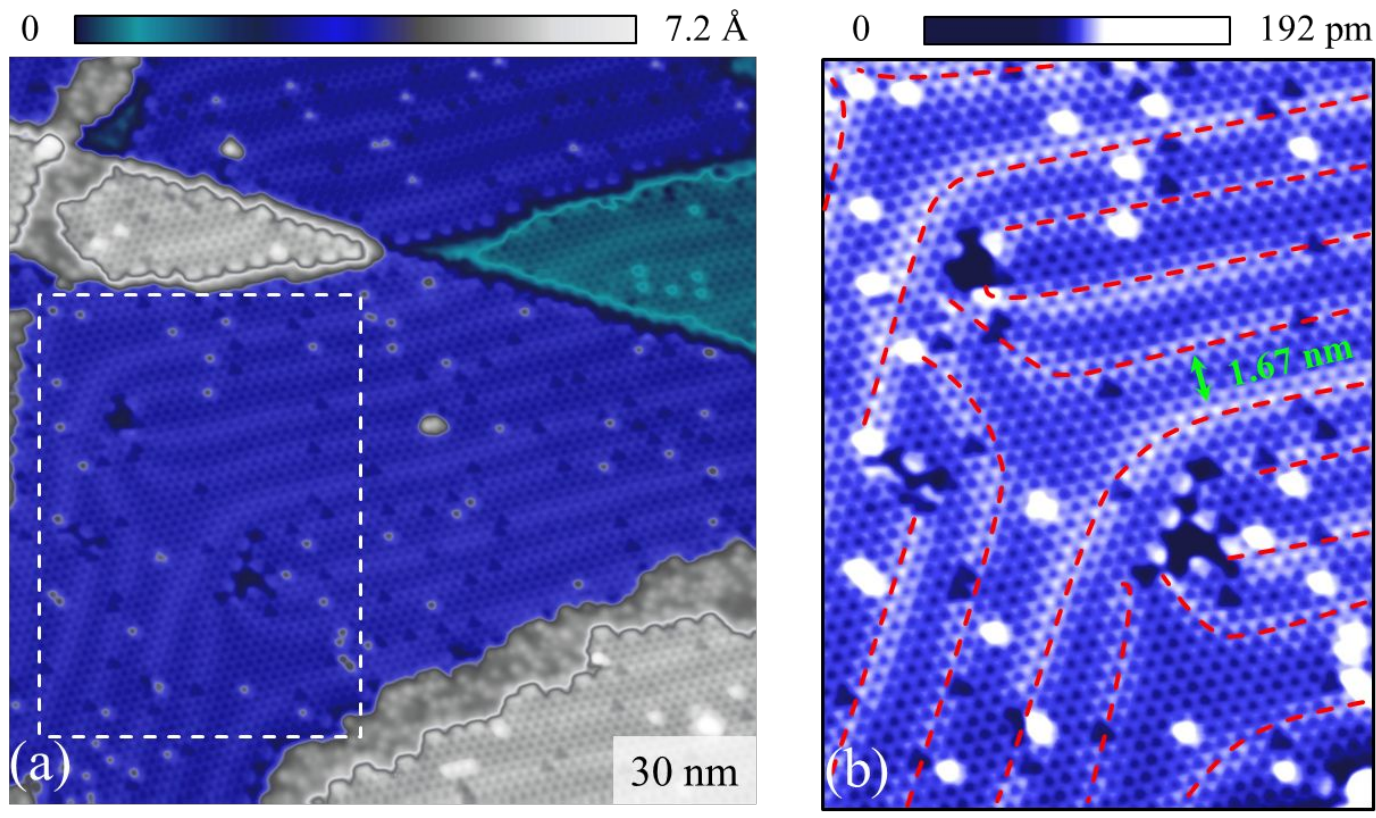

Figure S5 Topographic STM images showing different oriented modulations and

the domain-like structure. (a) Large scaled STM images. (b) Zoom-in high resolution image for selected area, which shows the QDWs can be aligned along other $\Gamma-\mathrm{M}$ directions.

Large scaled images for uniform long range CDW orders
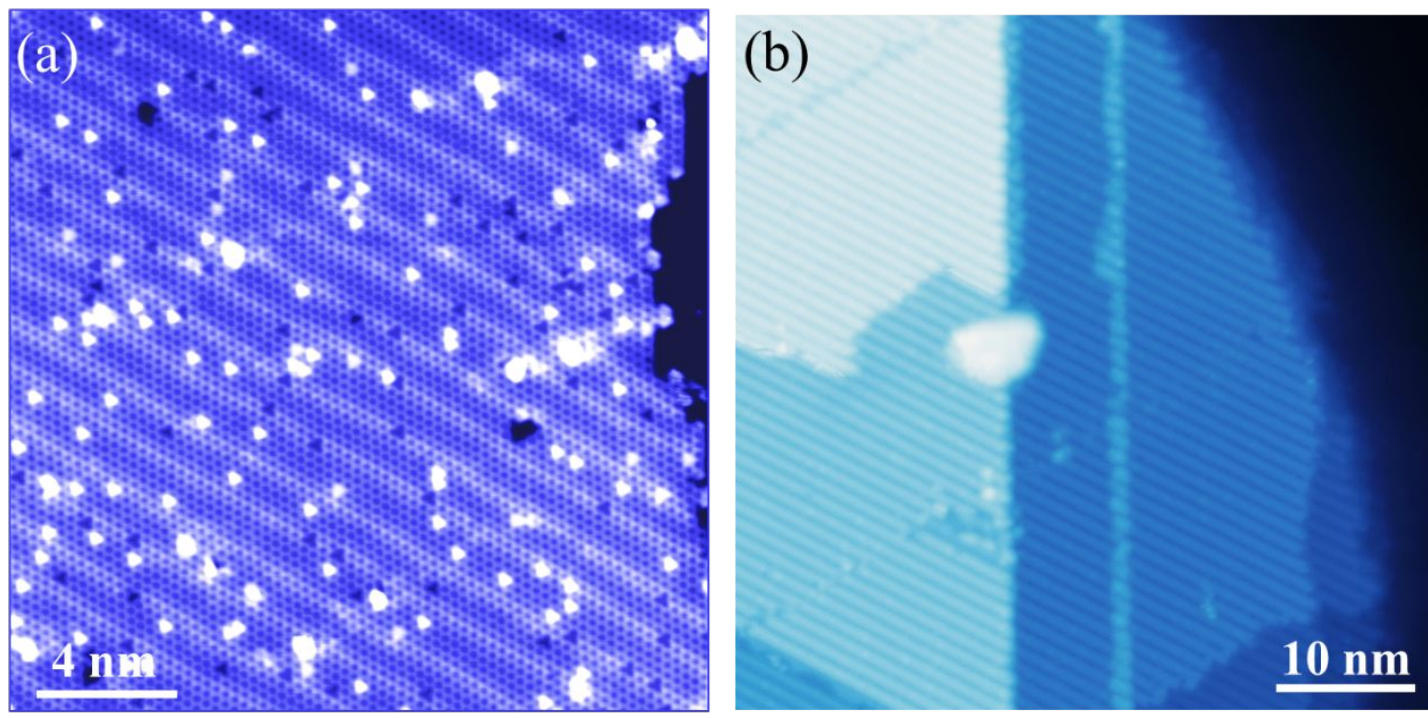

Figure S6 Large-scaled topographic STM images showing long range CDW orders. (a) Weak CDW in 2.7 at $\% \mathrm{Cr}-\mathrm{Mo}_{2} \mathrm{C}$ sample. The image size is $20 \times 20 \mathrm{~nm}^{2}$. (b) Strong CDW in 9.4 at $\% \mathrm{Cr}-\mathrm{Mo}_{2} \mathrm{C}$ sample. The image size is $50 \times 50 \mathrm{~nm}^{2}$. 
Topographic images for $\mathbf{2 . 7 \%} \mathrm{Cr}$-doped sample measured above and below Tc
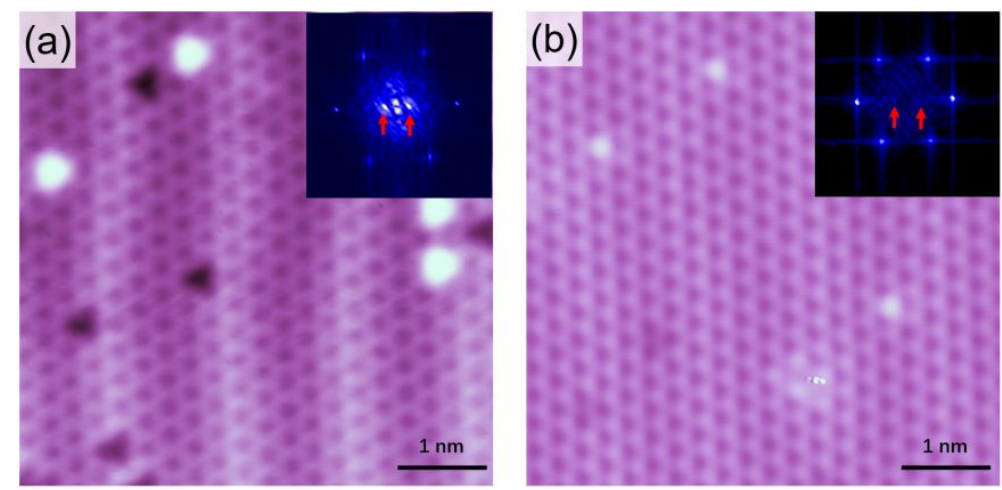

Figure S7. (a, b) show the STM images taken at the temperatures of $4.8 \mathrm{~K}$ (a) and 0.4 $\mathrm{K}$ (b) on $2.7 \%$ doped sample, respectively.

Resistance versus increasing and decreasing temperature

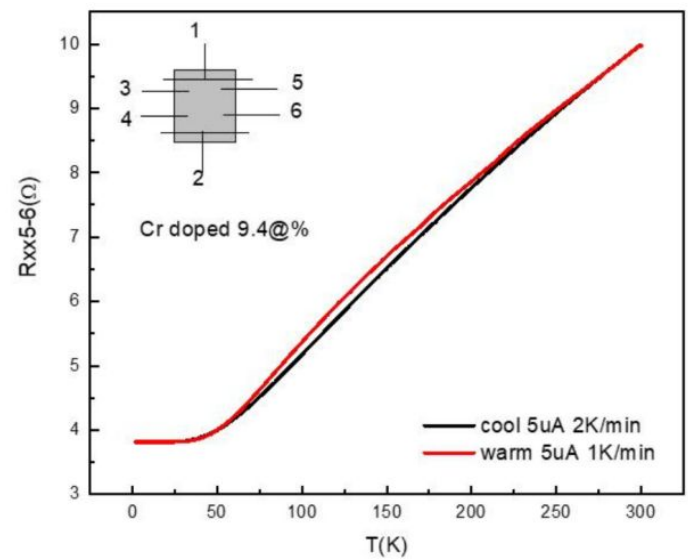

Figure S8. Resistance versus increasing (red) and decreasing (black) temperature.

Density waves and partially-opened asymmetric DW gaps observed in another group of samples. 

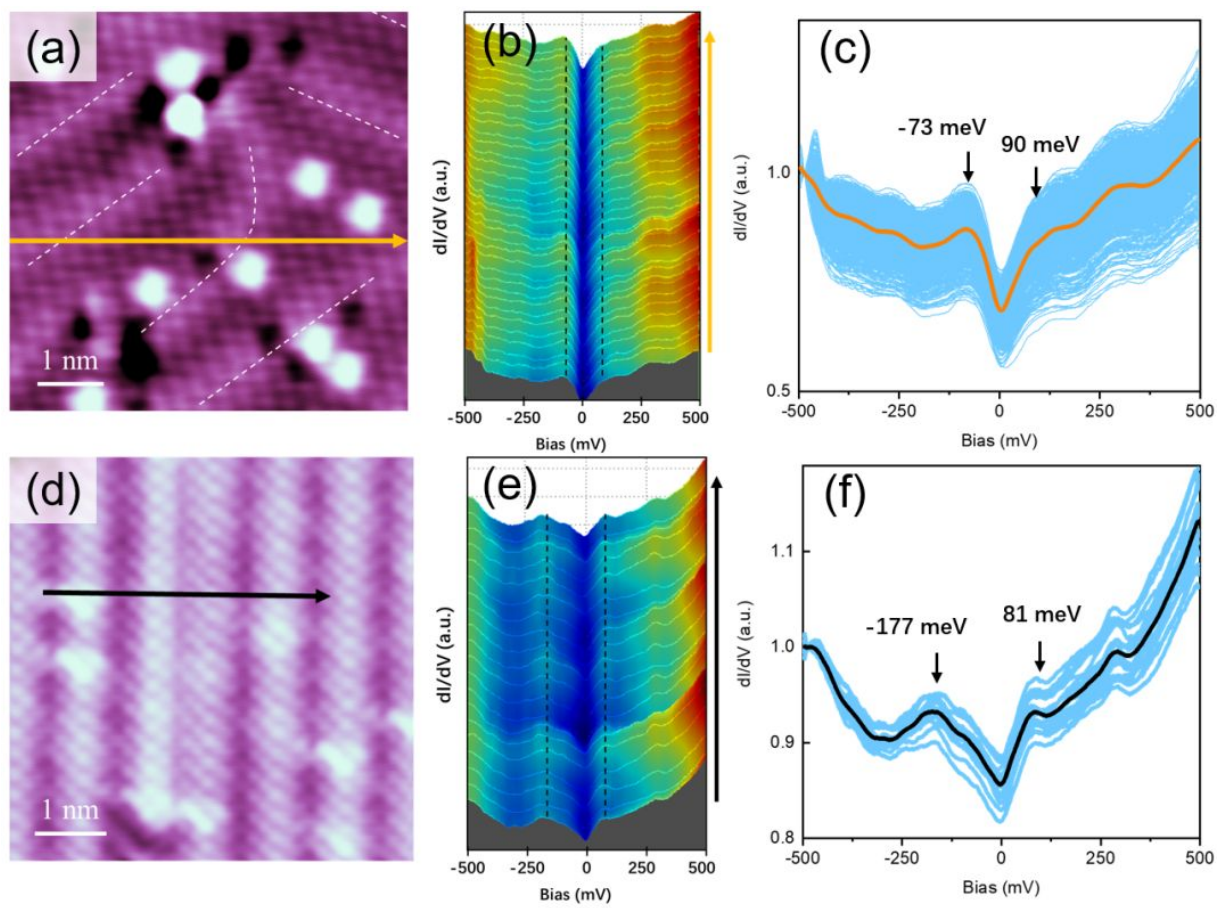

Figure S9. Density waves and partially-opened asymmetric DW gaps observed in another group of samples. (a) Topographic image of lightly $\mathrm{Cr}$ doped sample (2.7 at $\%$ ). The dotted white line indicates the 1D density wave in three equivalent directions. (b) Waterfall display of a set of spectra measured along the arrow line in (a). (c) Origin line: the average of 1225 spectra which are measured at points with a matrix of $35 \times 35$ uniformly distributed in the area of (a). (d-f) The same measurements as (a-c) conducted at 9.4 at $\%$ Cr doped sample. Averaged value of 20 spectra are shown in (f). 